\title{
Hepatitis B Virus among Dental Clinic Workers and the Risk Factors Contributing for its Infection
}

\author{
Al Kasem MA Abbas ${ }^{1}$, Abbas M Al-Kebsi ${ }^{2}$, Ebtihal M Madar ${ }^{1}$ and Hassan A Al-Shamahy ${ }^{3 *}$ \\ ${ }^{1}$ Department of Maxillo-Facial, Faculty of Dentistry, Sana'a University, Republic of Yemen \\ ${ }^{2}$ Department of Prosthodontics, Faculty of Dentistry, Sana'a University, Republic of Yemen
}

${ }^{3}$ Faculty of Dentistry, Sana'a University, Yemen

*Corresponding author: Hassan A Al-Shamahy, Faculty of dentistry, Medical Microbiology and Clinical Immunology, Faculty of Medicine and Health Sciences, Sana'a University, Republic of Yemen, PO Box 775 Sana'a, Yemen.

Received Date: October 01, 2018

Published Date: October 17, 2018

\begin{abstract}
Objectives: Dental clinic workers (DCWs) in Yemen have an additional risk of getting infected with HBV from their workplace and till now they are not routinely vaccinated against HBV infection. This study aimed to estimate prevalence of hepatitis B virus infection and associated risk factors among DCW in selected dental clinics in Sana'a city.

Study design: Data were acquired from a cross sectional survey conducted among DCWs in 2014 at the Faculty of Dentistry, Sana'a University, in Sana'a city. A proportionate to size random sample was drawn per DCW category. A structured questionnaire was used to collect data about socio-demographic characteristics and risk factors. ELISA was used to test sera for HBV markers.

Result: The study included 246 dentists and 263 dental assistants, the sero-prevalence of current hepatitis B virus infection was 6.1\%; while prevalence of life time exposure to hepatitis B virus infection was $18 \%$. Prevalence of needle stick injuries, exposure to skin and to mucous membranes were $45.6 \%, 26.5 \%$ and $25.3 \%$ respectively. Cuts were also common with $41.1 \%$ of participants reporting a cut in a period of one year preceding the survey. Regular use of gloves, face mask, and glasses were reported by $74.4 \%$, $28.3 \%$ and $25.3 \%$ of participants respectively. Only $30.1 \%$ of respondents were vaccinated against hepatitis B virus infection and $52.1 \%$ were susceptible and could potentially be protected through vaccination. Longer duration in service was associated with risk of infection (OR ranged from 2.2-2.65; P value $=0.002)$. Being assistant $(\mathrm{OR}=1.97$; $\mathrm{P}$ value $=0.001)$ was associated with a higher risk of hepatitis B virus infection.
\end{abstract}

Conclusion: The prevalence of current and life time exposure to HBV infection was high. Exposure to potentially infectious body fluids was high and yet only a small percentage of DCW were vaccinated, so there is need to vaccinate all DCWs as a matter of policy and ensure a safer work environment.

Keywords: HBV; DCWs; Risk factors; Sana'a city; Yemen

\section{Introduction}

Hepatitis B virus (HBV) infection has long been known as an occupational hazard among dentists [1-3]. Serological surveys have indicated that $10-30 \%$ of dentists have serological evidence of past or current HBV infection [4,5]. To reduce the risk of HBV infection, dental personnel have been not obligatory to receive immunization against HBV [6] plus to use barrier methods such as gloves to prevent blood-borne infections acquired during dental procedures [6].
By 1992, over $80 \%$ of US dentists and over $90 \%$ of British dentists and dental assistants reported HBV vaccination [7,8], while by 2014 in developing countries low rates were occurred e.g. Republic of Georgia, Nigeria, and South Africa the coverage rates were $12 \%, 64.5 \%$ and $67.9 \%$ respectively [8-10]. The use of barrier methods has generally increased over time in developed countries while in developing countries the uses of barrier methods are low until now. A 1993 UK study and 2014 German study indicated that 
$95 \%$ to $100 \%$ of dentists used gloves and from $83 \%$ to $100 \%$ used face masks $[11,12]$. A similarly high use of gloves was noted among US dentists $[13,14]$, although substantially lower rates of glove use were observed in Russian Federation [15] and Sweden [16].

The endemicity of infection was considered high in Yemen, where prevalence of positive HBsAg among adults ranged from $8 \%$ to $20 \%$, among infants was $4.1 \%$, and up to $50 \%$ of the population generally has serological evidence of previous HBV infection in old reports [17-20]. However, recent studies reported a lower rate of HBsAg, in which it ranges from $0.7-2 \%$ among the general population [21-24]. In the search for possible modes of cross-infection, HBsAg has been detected in nearly all body fluids of HBsAg carriers $[25,26]$. However, epidemiological and serological evidence implicates saliva as the most probable vehicle for spreading HBV among contacts without apparent percutaneous exposures to blood products. Accordingly, attention has focused on the risks of infection by dental care, either as a result of patientpatient exposures by inadequately sterilized instruments or of dentist-patient exposures by intimate contacts with HBsAg carriers $[25,26]$.

We present the results of a 2014 survey of dental personnel in Sana'a city, Yemen. The purposes of this survey were to evaluate what proportion of dentists and dental assistants had serological evidence of current or previous HBV infection, susceptibility rate to $\mathrm{HBV}$, what were their risk factors for exposure, what was their HBV vaccine coverage, and what was the frequency of use and effectiveness of barrier methods to prevent HBV infection.

\section{Subjects and Methods}

\section{Study area}

This cross-sectional epidemiological study was conducted among dental clinic personal care whom representative dental clinics in Sana'a city in Yemen.

\section{Study population and sample size}

This cross-sectional study was carried out starting in June and ending in August 2014. A consent form was filled by each participant. The sample size for the study was calculated as follow: First, the rate of HBV was considered among dental clinic personals in Sana'a city, difference (worst acceptable result higher or lower the true rate) and confidence interval as 3\%, 0.5\% and $99.9 \%$ respectively. Accordingly, a sample size of at least 458 subjects were required from the population of dental clinic personals in Sana'a city [roughly $5000 \mathrm{n}][27,28]$. The sample was selected by a systematic random method. All dental clinics in Sana'a city were listed [321 clinics), then by the use of a simple random selection, 120 of these dental clinics were selected; finally, all person working in the clinic were selected (about $2 \%$ of them who refused to participate in the study).

\section{Data collection}

All participants gave oral consent, completed a questionnaire, and had blood drawn for HBV serological testing. The questionnaires and blood samples were labeled with a study number which was also given to the study participants so that they could receive their test results by telephone. The questionnaire covered demographics, professional characteristics (type, duration, quantity of practice; patient characteristics; procedures performed), protective barrier measures used during dental procedures, HBV vaccination status, occupational exposures to blood and saliva, and other personal risk factors for hepatitis, such as blood transfusion, cupping, tattoo, etc.

\section{Laboratory tests}

Vein puncture of whole blood was collected; then sera were separated and tested for HBV markers by an Enzyme-linked Immunosorbant assay (ELISA) using a commercially available kit provided by Roche Diagnostics.

\section{Case definitions and data analysis}

All persons with anti-HBc were considered to have had serological signs of previous or current HBV infection. Anti-HBcpositive persons who also had HBsAg positive were identifying as HBV carriers. Persons with anti-HBs without anti-HBc were identifying to have been immunized against HBV. According to this definition, some persons who were vaccinated after they had previously been infected would be added up as not having been vaccinated. Persons with neither anti-HBs nor anti-HBc were considered to be HBV antibody negative (susceptible).

To relate possible risk factors for HBV infection, the data were examined in a case-control study format. For HBV, persons with evidence of previous or current infection with HBV (anti-HBcpositive) were matched up with those who were HBsAg negative and anti-HBc negative, and thus did not have serological evidence of previous HBV infection. Differences in categorical variables were assessed using Fisher's exact tests where appropriate. Ninetyfive percent confidence intervals for odds ratios were calculated according to the method of Cornfield and $95 \%$ confidence limits for simple proportions were calculated by an exact binomial method using EPI-INFO.

\section{Result}

Table 1: The prevalent rate and odds ratio (risks) of contracting HBV for different occupations, gender, practice setting and duration of the wok for DCWs, Sana'a city, Yemen.

\begin{tabular}{|c|c|c|c|c|}
\hline Factors & Infection n=123 N (\%) & Odds ratio & CI 95\% & PV \\
\hline Dentist n=246(48.3\%) & $44(17.9)$ & 0.51 & $0.33-0.79$ & 0.001 \\
\hline Dental assistants $\mathrm{n}=263(51.7 \%)$ & $79(30)$ & 1.97 & $1.3-3.1$ & 0.001 \\
\hline \multicolumn{2}{|c|}{ Gender } & 0.65 & $0.0 .41-1$ & 0.03 \\
\hline Male $\mathrm{n}=244(47.9 \%)$ & $49(20.1 \%)$ & 1.54 & $1-2.3$ & 0.03 \\
\hline Female $\mathrm{n}=265(52.1 \%)$ & $74(27.9 \%)$ &
\end{tabular}




\begin{tabular}{|c|c|c|c|c|}
\hline \multicolumn{3}{|c|}{ Practice setting } \\
\hline Private $\mathrm{n}=340(66.8 \%)$ & $85(25 \%)$ & 1.2 & $0.7-1.83$ & 0.52 \\
\hline Government $\mathrm{n}=169(33.2 \%)$ & $38(22.5 \%)$ & 0.87 & $0.6-1.4$ & 0.53 \\
\hline \multicolumn{5}{|c|}{ Duration of the practice } \\
\hline 5 years $\mathrm{n}=111(21.8 \%)$ & $15(13.5 \%)$ & Reference & \\
\hline$>10$ years $\mathrm{n}=224(44 \%)$ & $57(25.4 \%)$ & 2.2 & $1.13-4.72$ \\
\hline years $\mathrm{n}=174(34.2 \%)$ & $51(29.3 \%)$ & 2.65 & $1.35-5.3$ & 0.01 \\
\hline
\end{tabular}

A total of 509 DCWs; 246 dentists and 263 dental assistants were participated in the study. The demographic characteristics of the study sample are shown in Table 1 . Table 2 shows the interpretation of serological markers of HBV among dentists and dental assistant's participants in HBV study, Yemen. Among the enrolled dentists 44 (17.9\%) (OR=0.51, 95\% CI=0.33-0.79, $\mathrm{PV}=0.001$ ) had serological evidence of previous or current HBV infection while a higher significant rate and association among the enrolled dental assistants 79 (30\%), (OR=1.97, 95\% CI=1.3$3.1, \mathrm{PV}=0.001$ ) had serological evidence of previous or current HBV infection. Among the enrolled males 49 (20.1\%) (OR=0.65, 95\% CI=0.42-1, PV=0.03) had serological evidence of previous or current HBV infection while higher rate and association among the enrolled females 74 (27.9\%), (OR=1.54, 95\% CI=1 - 2.3, PV=0.03) had serological evidence of previous or current HBV infection (Table 3).

Table 2: Occupational possible risk factors for HBV among DCWs with previous and current HBV infection.

\begin{tabular}{|c|c|c|c|c|c|}
\hline Possible Risk Factors & Exposure n (\%) & Infection $n=123$ n (\%) & Odds Ratio & $\mathrm{Ci} 95 \%$ & pv \\
\hline Needle stick injuries & $232(45.6)$ & $93(40.1)$ & 5.5 & $3.4-8.98$ & $>0.001$ \\
\hline Cuts & $209(41.1)$ & $71(34 \%)$ & 2.5 & $1.6-3.79$ & $>0.001$ \\
\hline \multicolumn{6}{|c|}{ Contact with blood/saliva } \\
\hline On skin & $133(26.5)$ & $81(60.9)$ & 2.97 & $1.9-4.7$ & $>0.001$ \\
\hline On mucus membrane & $129(25.3)$ & 49 (37.9) & 2.53 & $1.6-4$ & $>0.001$ \\
\hline \multicolumn{6}{|c|}{ Consisting using Gloves } \\
\hline Yes & $379(74.4)$ & $92(24.3)$ & 1.02 & $0.6-1.7$ & 0.95 \\
\hline No & $130(25.6)$ & $31(23.8)$ & 0.98 & $0.6-1.6$ & 0.95 \\
\hline \multicolumn{6}{|c|}{ Glasses } \\
\hline Yes & $129(25.3)$ & $28(21.7)$ & 0.83 & $0.5-1.3$ & 0.45 \\
\hline No & $380(74.7)$ & $95(25)$ & 1.2 & $0.7-2$ & 0.45 \\
\hline \multicolumn{6}{|c|}{ Face mask } \\
\hline Yes & $144(28.3)$ & $37(25.7)$ & 1.12 & $0.7-1.79$ & 0.61 \\
\hline No & $365(71.7)$ & $86(23.5)$ & 0.89 & $0.6-1.4$ & 0.61 \\
\hline \multicolumn{6}{|c|}{ Vaccination to HBV } \\
\hline Yes & $153(30.1)$ & & 0.19 & $0.1-0.35$ & $>0.01$ \\
\hline No & $356(69.9)$ & & 5.3 & $2.7-10.6$ & \\
\hline
\end{tabular}

Table 3: General risk factors of contacting HBV among DCWs with previous and current HBV infection.

\begin{tabular}{|c|c|c|c|c|c|}
\hline & Exposure N (\%) & Infection n=123 N (\%) & Odds ratio & CI 95\% & PV \\
\hline Blood transfusion & 19 & 7 & 1.88 & $0.65-5.3$ & 0.18 \\
\hline Cupping & 3 & 1 & 1.57 & $0.28-6.9$ & 0.7 \\
\hline Travel abroad & 90 & 2 & 0.06 & $0.01-0.24$ & $>0.0001$ \\
\hline Tattooing & 9 & 1 & 0.39 & $0.02-3.1$ & 0.35 \\
\hline
\end{tabular}

Table 4: Interpretation of serological markers of HBV among dentists and dental assistant's participants, Sana'a city, Yemen, February 2014.

\begin{tabular}{|c|c|c|c|c|c|}
\hline \multicolumn{3}{|c|}{ Serological Markers } & Interpretation & Number \\
\cline { 1 - 3 } HBs Ag & Anti-HBs & Anti-HBC & Susceptible & 265 & 52.1 \\
\hline Negative & Negative & Negative & Immune after infection (previous infection) & 92 & 18 \\
\hline Negative & Positive & Positive & Immune after vaccination & 100 & 20 \\
\hline Negative & Positive & Negative & Current infection & 31 & 6.1 \\
\hline Positive & Negative & Positive & Indeterminate four possible & 21 & 4.1 \\
\hline Negative & Negative & Positive & & & 21 \\
\hline
\end{tabular}


1-Resolving infection (window phase)

2-Remote resolved infection with low anti-HBs

3-Chronic infection with low level of HBsAg

4-Flase positive anti-HBC hence susceptible

To determine the possible risk factors for HBV acquisition, the 123 DCWs with serological evidence of previous or current HBV infection (HBsAg and anti-HBc) were compared to the 365 without $\mathrm{HBsAg}$, anti-HBC and or anti-HBS antibody (excluding inteminante cases). There was a significance risk factors ( $\mathrm{P}>0.001)$ of needle stick injury (OR=5.5, 95\% CI=3.4-8.98), cuts (OR=2.5, 95\% CI=1.6 -3.79), saliva or blood exposure to skin $(\mathrm{OR}=2.97,95 \% \mathrm{CI}=1.9$ 4.7 ), and salvia or blood exposure to mucus membrane (OR=2..53,
95\% CI=1.6- 4) with serological evidence of previous or current HBV infection (Table 4). Nineteen DCWs reported having received a previous blood transfusion; 7 of whom had serological evidence of previous or current HBV infection. Only three DCWs reported cupping; one had evidence of previous HBV infection 90 travel abroad; two had evidence of previous HBV infection and 9 reported tattooing; one had evidence of previous HBV infection (Table 5).

Table 5: The reasons after DCWs not to take HBV vaccine.

\begin{tabular}{|c|c|c|}
\hline Reasons & Number & $\%$ \\
\hline Vaccination not offered & 161 & 45.2 \\
\hline Concern over vaccine safety & 42 & 12 \\
\hline Did not get around to obtaining vaccine & 99 & 28 \\
\hline HBV is not serious & 53 & 15 \\
\hline
\end{tabular}

Table 6: The methods of taking patients history by DCWs for the dental clinic visitors.

\begin{tabular}{|c|c|c|}
\hline \multicolumn{2}{|c|}{ Number } & \% \\
\hline \multicolumn{2}{|c|}{ Patients history type for infection (HBV, HCV, HIV etc) } \\
\hline Written only & 146 & 28.7 \\
\hline Oral only & 34 & 6.7 \\
\hline Written and oral & 83 & 16.3 \\
\hline None & 254 & 49.9 \\
\hline At first visit & Patients history frequency & 41.6 \\
\hline At certain intervals & 212 & 6.9 \\
\hline Each visit & 35 & 1.6 \\
\hline
\end{tabular}

Table 7: Demographic and professional characteristics of the HBV survey participants, Sana'a city, Yemen, February 2014.

\begin{tabular}{|c|c|c|c|c|}
\hline \multirow{2}{*}{ Characteristics } & \multicolumn{2}{|c|}{ Dentists n=246 } & \multicolumn{2}{|c|}{ Dental assistants $n=263$} \\
\hline & Number & $\%$ & Number & $\%$ \\
\hline \multicolumn{5}{|c|}{ Gender } \\
\hline Male & 132 & 53.7 & 112 & 42.6 \\
\hline Female & 114 & 46.3 & 151 & 57.4 \\
\hline \multicolumn{5}{|c|}{ Age groups } \\
\hline$<22$ years & 37 & 15 & 68 & 25.9 \\
\hline 23-27 years & 45 & 18.3 & 52 & 19.8 \\
\hline 28-32 years & 52 & 21.1 & 57 & 21.7 \\
\hline 33-37 years & 44 & 17.9 & 54 & 20.5 \\
\hline$\geq 38$ years & 68 & 27.6 & 32 & 12.2 \\
\hline \multicolumn{5}{|c|}{ Practice setting } \\
\hline Private setting & 165 & 67.1 & 175 & 66.5 \\
\hline Governmental clinic setting & 81 & 32.9 & 88 & 33.5 \\
\hline
\end{tabular}

Estimates of hepatitis B immunization coverage were obtained in two ways: via antibody testing and via self-report on the questionnaire. Only $20 \%$ of the study participants had serological evidence of previous immunization (Table 6). Among all participants, $30.1 \%$ reported having been immunized. Among the 353 DCWs who reported no previous immunization, the principal reasons stated for not having been vaccinated were the vaccination was not offered (45.2\%), concern over vaccine safety (12\%), did 
not get around to obtaining it (28\%), and that HBV is not so serious (15\%) (Table 7).

Over $78 \%$ of the DCWs reported that they obtain in their clinics from patients a risk factor history for hepatitis B, C and HIV mostly by written means and mostly only at the first visit (Table 7). A $74.4 \%$ of the DCWs reported consistent use of protective gloves. Only $25.3 \%$ consistent use of protective glasses and $28.3 \%$ consistent use of protective face mask (Table 4).

\section{Discussion}

Our results suggest that occupational transmission of HBV in dental settings occurs sometimes, and frequently. The finding that more than $6.1 \%$ of Sana'a city DCWs had HBsAg was opposing with prevalence of less than $2 \%$ found among general dentists in Taiwan [280] Japan [25] and North America [4, 291]. HBV vaccine coverage was suboptimal among the Sana'a DCWs surveyed, with approximately $70 \%$ of the Sana'a DCWs reporting not having been vaccinated. The proportion with serological evidence of HBV vaccination was even lower, presumably due to loss of antibody following complete or partial vaccination failure to produce antiHBs or due to inaccurate self- reporting. The self-reported HBV vaccination coverage among Sana'a DCW was lower than that observed in Germany and North America, Japan [7,8,25,29] and the United Kingdom [11]. Similar to other studies, dentists had higher coverage levels than assistants $[11,29,30]$. Among the Sana'a dentists without serological evidence of previous HBV vaccination, $24.1 \%$ had serological evidence of previous or current HBV infection, indicating that the contemporary risk of HBV acquisition remains high and similar to that observed in earlier North American studies $[2-4,7,29]$. This continuing high risk could be in part due to inconsistent use of or ineffectiveness of recommended barrier prevention measures to prevent transmission of blood-borne infections.

Among the Sana'a DCWs, the odds of previous or current HBV infection did not differ according to the consistent use of gloves, face masks or eye glasses, suggesting that these modalities had limited or no efficacy. Unfortunately, there are few other comparison data about the efficacy of barrier prevention measures. Two studies conducted during the 1980s showed no relationship between the use of gloves, face masks, or eye protection and previous HBV infection [31,32]. However, in one study, so few dentists used face masks that the power to detect a protective effect was low [32].

The conclusion that face masks may have reduced the risk of HBV infection must be interpreted with some caution as the small number of previously HBV infected dentists precluded a multivariate analysis to examine potential confounding factors. Nevertheless, the fact that only the use of face masks, but neither gloves nor eye glasses, had an apparent protective effect suggests that this finding was not merely due to general attention toward preventive measures or other general practice characteristics. In addition, this study only had an approximately $50 \%$ power to detect a fourfold reduction in the odds of previous infection by gloves or eye glasses. Nevertheless, the fact that the point estimates of the odds ratios were $1 \pm 1$ would suggest that no significant effect would have been found even with a much larger sample size.
Two general limitations of the study must be considered when interpreting the results the relatively small sample size and narrative data collection for vaccination history and using protective measures. Although the demographic characteristics of our study sample resembled that of all Sana'a DCW, the voluntary nature of the study may have introduced selection bias.

This study has several important implications for public health policy. First, despite a continuing high risk of HBV infection and the existence of longstanding recommendations for routine HBV vaccination, vaccine coverage among dentists and dental assistants are not to be adequate. Finally, given the high costs of gloves [13] and their apparent lack of efficacy in preventing HBV infection in this dictates further evaluation as to the cost-effectiveness of their routine use in dentistry. However, this cost-effectiveness evaluation has to take into account the potential for prevention of other bloodborne viruses, particularly HCV and HIV.

\section{Conclusion and Recommendations}

The prevalence of current and life time exposure to HBV infection was high. Exposure to potentially infectious body fluids was high and yet only a small percentage of DCWs were vaccinated, so there is need to vaccinate all DCWs as a matter of policy and ensure a safer work environment. Adequate funding by the Government is needed for HBV vaccination and legislation needs to be introduced for adult vaccination against $\mathrm{HBV}$ infection, especially for those at high risk. Also, the DCWs must receive comprehensive safety training in order to avoid unnecessary risk of blood-borne infections. Provision of required preventive devices (Engineered Sharps Injury Prevention Devices) is essential for following/ adopting universal precautions. Raising awareness of the risk of exposure among DCWs is needed also.

\section{References}

1. Feldman RE, Schiff ER (1975) Hepatitis in dental professionals. JAMA 232(12): 1228-1230.

2. Smith JL, Maynard JE, Berquiest KR (1976) From the Center for Disease Control: comparative risk of hepatitis B amono physicians and dentists. J Infect Dis 133(6): 705-706.

3. Mosley JW, Edwards VM, Casey G, Redeker A, White E (1975) Hepatitis B virus infection in dentists. N Engl J Med 293(15): 729-734.

4. Thomas DL, Gruninger SE, Siew C, Joy ED, Quinn TC (1996) Occupational risk of hepatitis $C$ infections among general dentists and oral surgeons in North America. Am J Med 100(1): 41-45.

5. Pido B, Kagimu M (2005) Prevalence of hepatitis B virus (HBV) infection among Makerere University medical students. Afr Health Sci 5(2): 93-98.

6. (2008) Centers for Disease Control and Prevention. Healthcare Personnel Vaccination Recommendations.

7. Cleveland JL, Siew C, Lockwood SA, Gruninger SE, Gooch BF, et al. (1996) Hepatitis B vaccination and infection among U.S. dentists, 1983 \pm 1992 . J Am Dent Assoc 127(9): 1385-1392.

8. Ogoina D, Pondei K, Adetunji B, Chima G, Isichei C, et al. (2014) Prevalence of hepatitis B vaccination among health care workers in Nigeria in 201112. Int J Occup Environ Med 5(1): 51-56.

9. Topuridze M, Butsashvili M, Kamkamidze G, Kajaia M, Morse D, et al. (2010) Barriers to hepatitis B vaccine coverage among healthcare workers in the Republic of Georgia: An international Perspective. Infect Control Hosp Epidemiol 31(2): 158-164.

10. Burnett RJ, François G, Mphahlele MJ, Mureithi JG, Africa PN, et al. (2011). Hepatitis B vaccination coverage in healthcare workers in Gauteng Province, South Africa. Vaccine 29(25): 4293-4297. 
11. Scully C, Griffiths M, Levers H, Blake C, Chartres L (1993) The control of cross-infection in UK clinical dentistry in the 1990s: immunization against hepatitis B. Br Dent J 174: 29-31.

12. Mutters NT, Hägele U, Hagenfeld D, Hellwig E, Frank U (2014) Compliance with infection control practices in an university hospital dental clinic. GMS Hyg Infect Control 9(3): 18-30.

13. Nash KD (1992) How infection control procedures are affecting dental practice today. J Am Dental Assoc 123(3): 67-73.

14. Puttaiah R, Miller K, Bedi DR, Shetty S, Almas K, et al. (2011) Comparison of knowledge, attitudes and practice of dental safety from eight countries at the turn of the century. J Contemp Dent Pract 12(1): 1-7.

15. Budnyak MA, Gurevich KG, Fabrikant KG, Miller K, Puttaiah R (2012) Dental infection control and occupational safety in the Russian Federation. J Contemp Dent Pract 13(5): 703-712.

16. Hellegran K (1994) Use of gloves among dentists in Sweden \pm a 3-year follow-up study. Swed Dent J 18(1-2): 9-14.

17. Al-Nassiri KA, Raja'a YA (2001) Hepatitis B infection in Yemenis in Sana'a: pattern and risk factors. East Mediterr Health J 7(1-2): 147-152.

18. Al-Shamahy H (2000) Prevalence of Hepatitis B surface antigen and Risk factors of HBV infection in samples of healthy mothers and their infants in Sana'a, Yemen. Ann Saudi Med 20(5-6): 464-467.

19. Al-Shamahy HA, IA Rabbad, Al-Hababy A (2003) Hepatitis B virus serum markers among pregnant women in Sana'a, Yemen. Ann Saudi Med 23(12): 87-89.

20. Murad EA, Babiker SM, Gasim GI, Rayis DI, Adam I (2013) Epidemiology of hepatitis $\mathrm{B}$ and hepatitis $\mathrm{C}$ virus infections in pregnant women in Sana'a, Yemen. BMC Pregnancy Childbirth 13: 127.

21. Alodini AQ (2012) Prevalence of Hepatitis B Virus and Hepatitis C Virus infections among blood donors at Al-Thawra Hospital Sana'a City-Yemen. YJ MS 6: 15-20.
22. Al-Waleedi AA, Khader YS (2012) Prevalence of hepatitis B and C infections and associated factors among blood donors in Aden city, Yemen. East Mediterr Health J 18(6): 624-629.

23. Al-Shamahy HA, Hanash SH, Rabbad IA, Al-Madhaji NM, Naser SM (2011) Hepatitis B Vaccine Coverage and the Immune Response in children under 10 years old in Sana'a Yemen. Sultan Qaboos Univ Med J 11(1): 77-82.

24. Gacche RN, Kaid AM (2012). Epidemiology of hepatitis B and C infections in Ibb city Yemen. Hept Mon 12(7): 460-462.

25. Nagao Y, Matsuoka H, Kawaguchi T, Ide T, Sata M (2008) HBV and HCV infection in Japanese dental care workers. Int J Mol Med 21(6): 791-799.

26. Puttaiah R, Shulman JD, Youngblood D, Bedi R, Tse E, et al. (2009) Sample infection control needs assessment survey data from eight countries. Int Dent J 59(5): 271-276.

27. (2013) Statistical Year, Central Statistical Organization (CSO), Ministry of Statistics and Programme Implementation.

28. Kwo MY, Hahn LJ, Hong CY, Kao JH, Chen DS (1993) Low prevalence of hepatitis $C$ virus among dentists in Taiwan. J Med Virol 40(1): 10-13.

29. Klein RS, Freeman, Taylor RE, Stevens CE (1991) Occupational risk for hepatitis C virus infection among New York City dentists. Lancet 338: 1539-1542.

30. McCarthy GM, MacDonald JK (1998) Improved compliance with recommended infection control practices in the dental office between 1994 and 1995. Am J Infect Control 26(1): 24-28.

31. Noble MA, Mathias RG, Gibson GB, Epstein JB (1991) Hepatitis B and HIV infections in dental professionals: effectiveness of infection control procedures. J Can Dent Assoc 57(1): 55-58.

32. Reingold AL, Kane MA, Hightower AW (1988) Failure of gloves and other protective devices to prevent transmission of hepatitis B virus to oral surgeons. JAMA 259(17): 2558-2560. 